\title{
Circuit Fault Location Based on Dynamic Bayesian Network
}

\author{
Zhao Liming ${ }^{1,2}$, Liu Heping ${ }^{1}$ \\ 1.School of Automation and Electrical Engineering, University of Science and Technology, Beijing, \\ China \\ 2.Institute of Information, Guangdong Ocean University, Zhanjiang, China
}

Keywords: Analog circuit; fault diagnosis; dynamic Bayesian network; feature selection

\begin{abstract}
To improve the accuracy of analog circuit fault diagnosis, on account of the problem that is difficult to obtain a high accuracy of the test results for a single model, based on combinatorial optimization theory, an analog circuit fault diagnosis model based on dynamic Bayesian network is proposed. Firstly, circuit fault features are extracted, and then hidden Markov model and least squares support vector machine are used to establish combination diagnosis model of analog circuit fault, and finally the simulation experiment is used to analyze the performance of combination models. The results show that compared to other analog circuit fault diagnosis models, the proposed model not only improves the accuracy of analog circuit fault detection, but also has faster speed of fault diagnosis.
\end{abstract}

\section{Introduction}

With the development of ultra-large-scale integrated circuits and increasingly complex analog circuits, because of electric parameter drifts and non-linear characteristics, there are numerous causes for the occurrence of analog circuit fault, and the analog circuit fault diagnosis has been the difficult problems in mixed-signal circuit fault diagnosis [1]. Analog circuit fault diagnosis is essentially a problem of pattern classification, including two key steps of feature extraction and classifier design [2]. Feature extraction is the first step in an analog circuit fault diagnosis, because of the complexity of the analog circuit itself, as well as impacts of a variety of external factors, it is featured by nonlinear change. There is currently a lot of methods for analog circuits feature extraction, such as wavelet analysis, Volterra series methods [2], in which Volterra series can better capture the nonlinearity of circuit signal change with certain advantages, thus this paper employs the extracted fault features of analog circuit. The most original analog circuit fault diagnosis classifier is established by multiple linear regression method, but it is difficult to establish accurate fault classification model, and the correctness and timeliness of diagnosis results cannot meet the actual needs of the project [3]. Hidden Markov Model (HMM) has outstanding capabilities of pattern classification and has been widely used [3] in fault diagnosis. In recent years, with the development of nonlinear theory, among it, the neural network is most representative with outstanding nonlinear fitting ability. Analog circuit fault diagnosis has been successfully applied, but because the neural network is a kind of machine learning algorithms based on empirical risk minimization principle, there exist defects such as a large number of training samples, easily occurred over-fitting and others, and its applications in fault diagnosis are affected [3]. Support vector machine is excellent in performance, but the computational complexity of the training process and low efficiency cannot meet the real-time requirements of analog circuit fault diagnosis. In allusion to the problems of complexity in analog circuit fault and unavailable test results with high accuracy for single model, based on combinatorial optimization theory, this paper has proposed a combined analog circuit fault diagnosis model based on dynamic Bayesian model. Firstly, features of circuit fault are extracted, and then the combined analog circuit fault diagnosis model is established using dynamic Bayesian model, and finally the diagnostic performances of the model are analyzed by simulation experiments. 


\section{Extracting features}

In 1880, Italian mathematician Vito Volterra proposed concept of Volterra series, and as for nonlinear systems, he could recommend the transfer function similar to linear systems. Firstly, the energy values of Volterra series sequence h1 (m), h2 (m1, m2), h3 (m1, m2, m3) and h4 (m1, m2, $\mathrm{m} 3, \mathrm{~m} 4)$ are calculated and taken as fault features of circuit to be diagnosed [4].

$$
\left\{\begin{array}{l}
E_{1}=\sum_{m}\left|h_{1}(m)\right|^{2} \\
E_{2}=\sum_{m_{1}, m_{2}}\left|h_{2}\left(m_{1}, m_{2}\right)\right|^{2} \\
E_{3}=\sum_{m_{1}, m_{2}, m_{3}}\left|h_{2}\left(m_{1}, m_{2}, m_{3}\right)\right|^{2} \\
E_{4}=\sum_{m_{1}, m_{2}, m_{3}, m_{4}}\left|h_{2}\left(m_{1}, m_{2}, m_{3}, m_{4}\right)\right|^{2}
\end{array}\right.
$$

When the circuit to be diagnosed fails, because there will be changes in the sequence all with Volterra series, and corresponding changes in E1 $\sim \mathrm{E} 4$, and the situation of changes in E1 $\sim \mathrm{E} 4$ and the circumstance of fault changing E1 $\sim$ E4 corresponds, thus energy values E1 $\sim$ E4 of $1 \sim 4$-order sequence all with Volterra series can be taken as fault features of circuits to be diagnosed.

\section{Fault classification principle of dynamic Bayesian}

As a dynamic signal time-series statistical model, dynamic Bayesian network is evolved based on Bayesian. It consists of two random processes, observation value sequence and status value sequence, ideal for continuous or discrete dynamic signal processing [2]. The model overcomes the weakness that the sensor data is diagnosed only by one point measured in traditional BIT fault diagnosis method, but it has made full use of dynamic time feature information before and after the failure. At the same time, dynamic Bayesian model also has a strong learning ability, able to acquire knowledge of experience through learning and training, thus classifying the unknown fault state to effectively avoid false alarms.

Dynamic Bayesian model can be specifically described as $\lambda=(\pi, A, B)$ : Among it, $\pi$ is the vector of the initial probability distribution; $A$ is for the transition probability matrix at initial state; $\pi, A$ describes that the output generated by the dynamic Bayesian model is state value sequence; $B$ is the initial observation probability matrix, describing a random process, and the output is observation value sequence. There are two basic algorithms for Dynamic Bayesian model: Baum-welch algorithm and forward - backward algorithm.

Baum-welch algorithm: through a set sequence of observations $O=\left(o_{1}, o_{2}, \cdots, o_{n}\right)$, in which $n$ is the measured length of observation value sequence, $\lambda$ is calculated using the maximum likelihood rule, so that $P(O / \lambda)$ can reach the maximum to obtain dynamic Bayesian model. It has mainly solves the training problem of dynamic Bayesian model. That is, the historical fault samples are given to produce dynamic Bayesian model by the algorithm and to complete learning and training.

(2) Forward - backward algorithm: $P(O / \lambda)$ is calculated on the basis of the given observation sequence $O$ and HMM model $\lambda$. It mainly solves the decision-making problem of dynamic Bayesian model. Namely, fault status classification of unknown observation sequence is made through well-trained dynamic Bayesian model.

Dynamic Bayesian model theory is shown in Figure 1. 


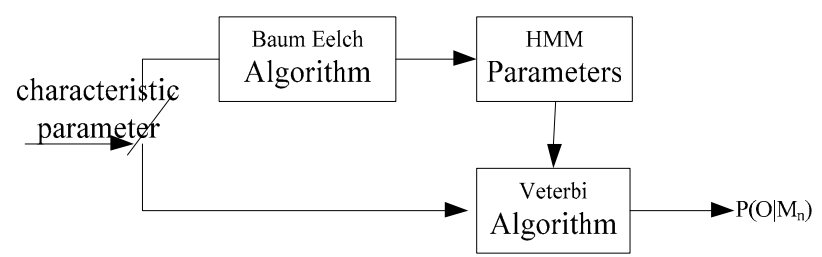

Figure 1 Working principle of dynamic Bayesian

\section{Classification principle of least square support vector machine}

Analog circuit fault diagnosis is essentially a problem of classification and regards the extracted feature vectors as the input vectors of dynamic Bayesian model to establish analog circuit fault diagnosis device. Assuming a training set is $\{\mathrm{xi}, \mathrm{yi}\}$, linear classification function of dynamic Bayesian model is:

$$
f(x)=w^{T} \varphi(x)+b
$$

In the formula, $\omega$ is the weight vector, and $b$ is the offset value [15].

According to the structure risk minimization principle, the formula (2) may represent a constrained optimization problem:

$$
\begin{aligned}
& \min _{w, b, e} J(w, e)=\frac{1}{2} W^{T} W+\frac{C}{2} \sum_{i=1}^{n} e_{i}^{2} \\
& \text { s.t. } \\
& y_{i}=w^{T} \varphi(x)+b+e_{i}, i=1,2, \cdots, l
\end{aligned}
$$

In the formula, $\mathrm{C}$ is penalty parameter; ei is prediction error.

By introducing Lagrange multipliers, the above constrained optimization problem turns into unconstrained dual space optimization to build Lagrange function

$$
L(w, b, e, \alpha)=J(w, e)-\sum_{i=1}^{l} \alpha_{i}\left(w^{T} \varphi\left(x_{i}\right)+b+e_{i}-y_{i}\right)
$$

In the formula, $\alpha_{i}$ is the Lagrange multiplier [15].

According KKT conditions, it can be obtained that

$$
\left[\begin{array}{cc}
0 & e 1^{T} \\
e 1 & Q+C^{-1} I
\end{array}\right]\left[\begin{array}{l}
b \\
a
\end{array}\right]=\left[\begin{array}{l}
0 \\
y
\end{array}\right]
$$

Among it

$$
\left\{\begin{array}{l}
y=\left(y_{1}, y_{2}, \cdots, y_{l}\right)^{T} \\
e 1=(1,1, \cdots, 1)^{T} \\
a=\left(a_{1}, a_{2}, \cdots, a_{l}\right)^{T}
\end{array}\right.
$$

In the formula, I represents unit matrix, $Q_{i j}=\left(\varphi\left(x_{i}\right) \varphi\left(x_{j}\right)\right)$

The radial basis kernel function is selected as the kernel function of dynamic Bayesian model, and the decision function of analog circuit fault diagnosis of dynamic Bayesian model is:

$$
f(x)=\operatorname{sgn}\left(\sum_{i=1}^{n} \alpha_{i} \exp \left(-\frac{\left\|x_{i}-x_{j}\right\|^{2}}{2 \sigma^{2}}\right)+b\right)
$$

In the formula, $\sigma$ is the width parameter of the radial basis kernel function.

Dynamic Bayesian model is binary classifier, while analog circuit fault diagnosis is a problem of multi-classification, and multi-classifier must be constructed to perform automatic image classification, established by adopting approach of "one-to-many", specifically as shown in Figure 2 . 


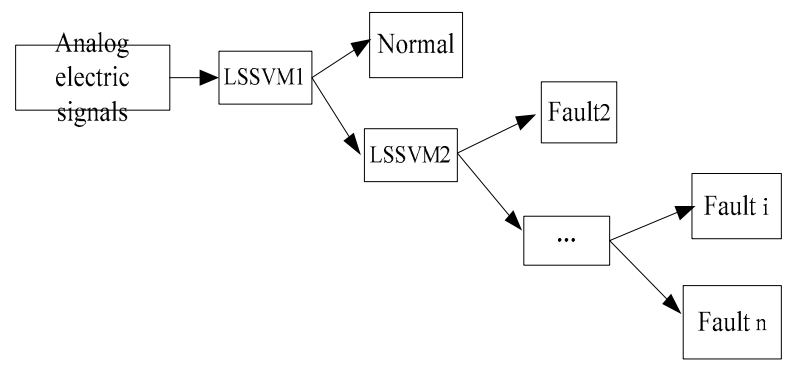

Figure 2 Construction of multi-classification dynamic Bayesian model

\section{Fault diagnosis process of dynamic Bayesian model}

Dynamic Bayesian model can establish analog circuit fault diagnosis model based on the temporal relations of signal circuit, able to approximate signal change trend within the category. But it determines the weakness only with the greatest similarity, based on risk minimization principle of separable problems, dynamic Bayesian model can make classifications, which has better overcome the deficiency existing in dynamic Bayesian model. Based on the theory of combinatorial optimization, the advantages of the dynamic Bayesian model and feature selection are fully used to overcome their shortcomings to form an analog circuit fault diagnostor with more optimal classification performance. The specific steps are as follows:

(1) Volterra-series energy value of the system is calculated to determine fault characteristics of the circuit and to establish training samples;

(2) The training samples are input into dynamic Bayesian model classifier for training to calculate the kernel function and parameters;

(3) Identification and diagnosis of analog circuit fault are conducted using the trained classifier of dynamic Bayesian model.

Analog circuit fault diagnosis process based on dynamic Bayesian model is shown in Figure 3.

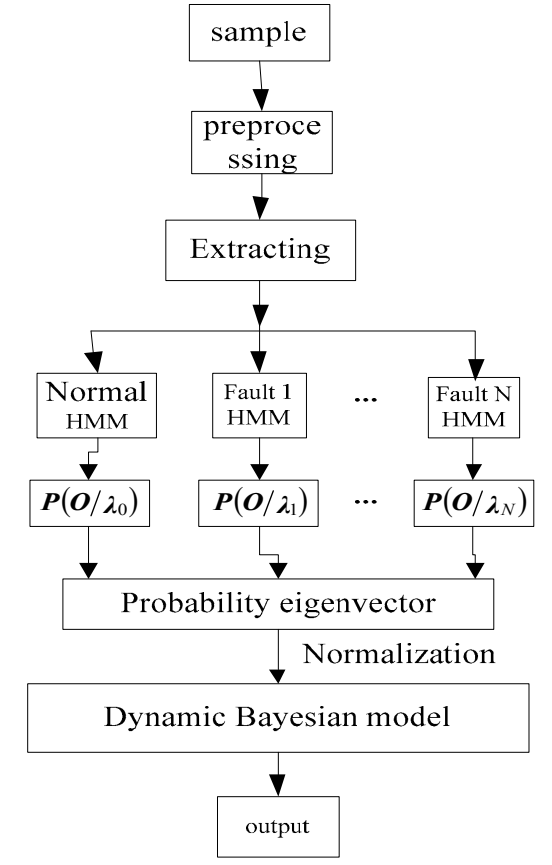

Figure 3 Analog circuit fault diagnosis flowchart 


\section{Simulation analysis}

\section{Simulation object.}

To test the performance of dynamic Bayesian model, the analog circuit in FIG. 4 is used as an example to conduct simulation experiments, and at the same time, single dynamic Bayesian model is selected to conduct simulation comparison experiment under the same conditions to test the rationality and superiority of dynamic Bayesian model. Supposed that there are six kinds of soft failures, the specific is as shown in Table 1.

Table 1 Circuit fault set

\begin{tabular}{ll}
\hline Fault No. & Fault type \\
\hline 1 & $\mathrm{R}_{2}^{+}(110 \mathrm{k}, 120 \mathrm{k})$ \\
2 & $\mathrm{R}_{2}^{-}:(80 \mathrm{k}, 90 \mathrm{k})$ \\
3 & $\mathrm{R}_{3}^{+}:(120 \mathrm{k}, 130 \mathrm{k})$ \\
4 & $\mathrm{R}_{3}^{-}(70 \mathrm{k}, 80 \mathrm{k})$ \\
5 & $\mathrm{C}_{1}^{+}(22 \mathrm{n}, 24 \mathrm{n})$ \\
6 & $\alpha_{1}^{+}(110,120)$ \\
\hline
\end{tabular}

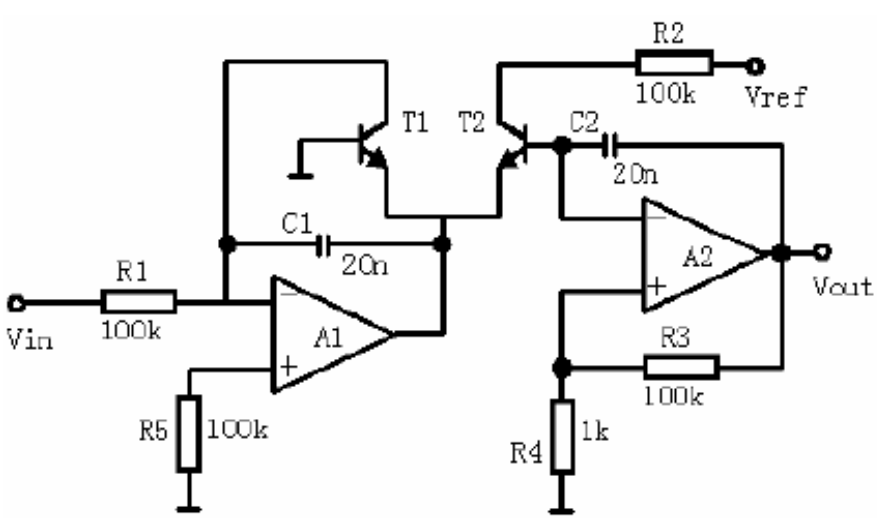

Figure 4 Simulation testing circuit

Feature normalization treatment

Assume a set of feature vector as $[\mathrm{x} 1, \mathrm{x} 2, \ldots, \mathrm{xn}]$, and moralize the feature to $[0,1]$ interval through formula (8) .

$$
\hat{x}=\frac{x_{i}-x_{\min }}{x_{\max }-x_{\min }}
$$

In which, $x \max$ is the maximum value, and $x \min$ is the minimum value.

\section{Results and analysis.}

1) Contrast of diagnosis accuracy rate

The accuracy rates of the simulation circuit fault diagnosis results of Bayesian model, dynamic Bayesian model and common feature selection are as shown in Table 2. From Table 2 it can be seen that the fault diagnosis average accuracy recognition rates with respect of Bayesian model, dynamic Bayesian model and common feature selection are separately improved by $7.42 \%$ and $3.70 \%$; because the simulation circuit has been comprehensively influenced by various factors during the work process, it has the non-liner changing features, it is difficult to establish the accurate fault diagnosis model by adopting the single Bayesian model and dynamic Bayesian model, while the dynamic Bayesian model fully makes use of the advantages of the feature selection and dynamic Bayesian model to dig the simulation circuit fault changing features from different aspects, it can more accurately describe the dynamic changing regulations of the simulation circuit, and improve the simulation circuit fault diagnosis, and obtain the satisfying diagnosis results. 
Table 2 Accuracy recognition rate $(\%)$ of different algorithms

\begin{tabular}{|c|c|c|c|c|}
\hline Fault pattern & Bayesian type & $\begin{array}{l}\text { Common fea- } \\
\text { ture selection }\end{array}$ & $\begin{array}{l}\text { - Dynamic } \\
\text { model }\end{array}$ & Bayesian \\
\hline No fault & 89.54 & 93.26 & 96.96 & \\
\hline $\mathrm{R}^{2+}$ & 89.54 & 93.26 & 96.96 & \\
\hline $\mathrm{R}^{2-}$ & 89.54 & 93.26 & 96.96 & \\
\hline $\mathrm{R}^{3+}$ & 89.54 & 93.26 & 96.96 & \\
\hline $\mathrm{R}^{3-}$ & 89.54 & 93.26 & 96.96 & \\
\hline $\mathrm{C}_{1}^{+}$ & 89.54 & 93.26 & 96.96 & \\
\hline$\alpha_{1}^{+}$ & 89.54 & 93.26 & 96.96 & \\
\hline $\begin{array}{l}\text { Average recog } \\
\text { nition rate }\end{array}$ & 89.54 & 93.26 & 96.96 & \\
\hline
\end{tabular}

2) Contrast of diagnosis speed

A good fault diagnosis method not only requires higher accurate recognition rate, but also requires better real-time, hence we test the same sample through the simulation, and sum up the fault diagnosis test time, and the results are see as in Table 3. From Table 3 it can be seen that, the time cost of fault diagnosis with respect of the single dynamic Bayesian model greatly decreases, and the modeling speed significantly accelerates, which is mainly because that the dynamic Bayesian model using its maximum similarity matching to reduce the unnecessary burdens of the Bayesian model, and greatly reduce the training time and improve the fault diagnosis speed.

Table 3 Time cost (s)contrast of different algorithm fault diagnosis

\begin{tabular}{|c|c|c|c|}
\hline Fault pattern & LSSVM & HMM & HMM-LSSVM \\
\hline No fault & 4.89 & 3.30 & 3.20 \\
\hline $\mathrm{R}^{2+}$ & 5.09 & 3.95 & 2.88 \\
\hline $\mathrm{R}^{2-}$ & 4.58 & 4.50 & 3.58 \\
\hline $\mathrm{R}^{3+}$ & 5.45 & 3.87 & 3.46 \\
\hline $\mathrm{R}^{3-}$ & 4.78 & 4.02 & 3.31 \\
\hline $\mathrm{C}_{1}^{+}$ & 5.53 & 4.58 & 3.16 \\
\hline$\alpha_{1}^{+}$ & 4.17 & 3.48 & 3.15 \\
\hline $\begin{array}{l}\text { Average tim } \\
\text { cost }\end{array}$ & 4.93 & 3.96 & 3.25 \\
\hline
\end{tabular}

\section{Conclusion}

In order to improve the accuracy rate of the simulation circuit fault diagnosis, a simulation circuit fault diagnosis model of dynamic Bayesian model is put forward. It firstly extracts the features of the fault diagnosis at Volterra progression, and adopts the Bayesian model to perform the primary diagnosis to the simulation circuit faults, and then adopts the dynamic Bayesian model to perform the further diagnosis to the simulation circuit faults, the simulation results show that, with the respect with the dynamic Bayesian model simulation circuit fault diagnosis model, the dynamic Bayesian model not only improves the accuracy rate of simulation circuit fault diagnosis, but also accelerates the fault diagnosis speed, and owns better real-time, which has wide application prospects in the simulation circuit fault diagnosis.

\section{Reference}

[1] Jie He, Yishuang Geng, Yadong Wan, Shen Li, Kaveh Pahlavan, A cyber physical test-bed for virtualization of RF access environment for body sensor network, IEEE Sensor Journal, 13(10), 3826-3836, Oct. 2013

[2] Wenhua Huang, Yishuang Geng, Identification Method of Attack Path Based on Immune 
Intrusion Detection, Journal of Networks, 9(4), 964-971, Jan. 2014

[3] Guanqun Bao, Liang Mi, Yishuang Geng, Mingda Zhou, Kaveh Pahlavan, A video-based speed estimation technique for localizing the wireless capsule endoscope inside gastrointestinal tract, 2014 36th Annual International Conference of the IEEE Engineering in Medicine and Biology Society (EMBC), Aug. 2014

[4] Degui Zeng, Yishuang Geng, Content distribution mechanism in mobile P2P network, Journal of Networks, 9(5), 1229-1236, Jan. 2014

[5] Mingda Zhou, Guanqun Bao, Yishuang Geng, Bader Alkandari, Xiaoxi Li, Polyp detection and radius measurement in small intestine using video capsule endoscopy, 2014 7th International Conference on Biomedical Engineering and Informatics (BMEI), Oct. 2014 